\title{
Risk of drug-induced interstitial lung disease in hospitalised patients: a nested case-control study
}

\author{
Taisuke Jo, ${ }^{1,2}$ Nobuaki Michihata, ${ }^{2}$ Hayato Yamana, ${ }^{2}$ Kojiro Morita, ${ }^{3,4}$ Miho Ishimaru, ${ }^{3,4}$ \\ Yasuhiro Yamauchi, ${ }^{1}$ Wakae Hasegawa, ${ }^{1}$ Hirokazu Urushiyama, ${ }^{1}$ Kazuaki Uda, ${ }^{4}$ \\ Hiroki Matsui, ${ }^{4}$ Kiyohide Fushimi, ${ }^{5}$ Hideo Yasunaga, ${ }^{4}$ Takahide Nagase ${ }^{1}$
}

\begin{abstract}
- Additional material is published online only. To view please visit the journal online (http://dx.doi.org/10.1136/ thoraxjnl-2020-215824)
\end{abstract}

'Department of Respiratory Medicine, Graduate School of Medicine, The University of Tokyo, Tokyo, Japan

2Department of Health Services Research, Graduate School of Medicine, The University of Tokyo, Tokyo, Japan

${ }^{3}$ Department of Health Services Research, Faculty of Medicine, University of Tsukuba, Tsukuba, Ibaraki, Japan

${ }^{4}$ Department of Clinical Epidemiology and Health Economics, School of Public Health, The University of Tokyo, Tokyo, Japan

${ }^{5}$ Department of Health Policy and Informatics, Tokyo Medical and Dental University Graduate School of Medicine, Tokyo, Japan

\section{Correspondence to} Dr Taisuke Jo, Department of Respiratory Medicine, Graduate School of Medicine, The University of Tokyo, Tokyo, Japan; jo-taisuke@umin.ac.jp

Received 18 July 2020 Revised 1 March 2021 Accepted 23 March 2021 Published Online First 22 April 2021

\section{Linked}

- http://dx.doi.org/10.1136/ thoraxjnl-2021-217373

\section{Check for updates}

(C) Author(s) (or their employer(s)) 2021. No commercial re-use. See rights and permissions. Published by BMJ.

To cite: Jo T, Michihata N, Yamana $\mathrm{H}$, et al. Thorax 2021;76:1193-1199.

\section{ABSTRACT}

Introduction Information on drug-induced interstitial lung disease (DILD) is limited due to its low incidence. This study investigated the frequencies of drug categories with potential risk in patients developing DILD during hospitalisation and analysed the risk of developing DILD associated with each of these drugs.

Methods Using a Japanese national inpatient database, we identified patients without interstitial pneumonia on admission who developed DILD and required corticosteroid therapy during hospitalisation from July 2010 to March 2016. We conducted a nested case-control study; four controls from the entire non-DILD patient cohort were matched to each DILD case on age, sex, main diagnosis, admission year and hospital. We defined 42 classified categories of drugs with 216 generic names as drugs with potential risk of DILD, and we identified the use of these drugs during hospitalisation for each patient. We analysed the association between each drug category and DILD development using conditional logistic regression analyses.

Results We retrospectively identified 2342 patients who developed DILD. After one-to-four case-control matching, 1541 case patients were matched with 5677 control patients. Six drug categories were significantly associated with the increased occurrence of DILD. These included epidermal growth factor receptor inhibitors (OR: $16.84,95 \%$ CI 9.32 to 30.41 ) and class III antiarrhythmic drugs (OR: 7.01, 95\% Cl 3.86 to 12.73). Statins were associated with reduced risk of DILD (OR: $0.68,95 \% \mathrm{Cl} 0.50$ to 0.92 ).

Conclusions We demonstrated significant associations between various drug categories and DILD. Our findings provide useful information on drug categories with potential risk to help physicians prevent and treat DILD.

\section{INTRODUCTION}

Lung injury is an increasingly common cause of morbidity and mortality in patients treated with either cytotoxic or non-cytotoxic drugs. Studies have reported various drugs that potentially cause drug-induced interstitial lung disease (DILD), including chemotherapeutic agents, antibiotics, antiarrhythmic agents and immunosuppressants. ${ }^{1-6}$ Corticosteroids are used for treating lung injury following DILD. ${ }^{1-11}$

Identifying a specific drug with potential risk for each patient who develops DILD is challenging due to the rarity of the disease. Although many studies

\section{Key messages}

What is the key question?

- Which drugs were associated with a higher risk of drug-induced interstitial lung disease?

What is the bottom line?

- Among the drugs with potential risk of druginduced interstitial lung disease, epidermal growth factor receptor inhibitors and class III antiarrhythmic drugs are highly likely to lead to the development of drug-induced interstitial lung disease.

Why read on?

- This study is the first to evaluate the risk of developing drug-induced interstitial lung disease associated with multiple drugs with potential risk.

have presented case report and case series data, these findings cannot clarify causal relationships between drugs and interstitial lung disease (ILD) due to the lack of a control group. One previous cohort study reported drugs associated with acute lung injury, ${ }^{6}$ but the generalisability of that study's results may be limited due to the small sample size. Difficulties in identifying drugs with potential risk of DILD may also be attributed to the ineffectiveness of laboratory testing, including drug lymphocyte stimulation test. ${ }^{1213}$

The present study aimed to measure the frequencies of drug categories with potential risk of DILD among patients who developed DILD during hospitalisation and to conduct a nested case-control study to evaluate the risk of developing DILD associated with each drug, using a Japanese national inpatient database.

\section{MATERIALS AND METHODS Data collection}

We obtained data from the Diagnosis Procedure Combination Database, which is a nationwide inpatient database in Japan. Data on discharge abstracts and administrative claims are collected from approximately seven million inpatients per year in around 1200 hospitals throughout Japan. The database includes main diagnoses, primary diagnosis on admission, comorbidities on admission and complications during hospitalisation, all of which 
are recorded by attending physicians using International Classification of Diseases and Related Health Problems 10th Revision (ICD-10) codes. The database also contains detailed information on age, sex, body mass index, activities of daily living, smoking status, medication, interventional procedures, surgical procedures and discharge status including in-hospital mortality.

\section{Identification of cases}

We conducted a nested case-control study. In the first step, we identified inpatients who developed DILD after admission from 1 July 2010 to 31 March 2016. We excluded patients who were diagnosed with DILD or ILD on admission. DILD was defined using the following ICD-10 codes: J702 (acute drug-induced interstitial lung disorders), J703 (chronic druginduced interstitial lung disorders) and J704 (drug-induced interstitial lung disorders, unspecified). ILD was defined using the following ICD-10 codes: B221 (HIV interstitial pneumonia), J700 (radiation pneumonitis), J701 (chronic and other pulmonary symptoms due to radiation), J841 (other ILDs with pulmonary fibrosis), J848 (other specified ILDs), J849 (ILD, unspecified), J990 (rheumatoid lung disease), J991 (respiratory disorders in other diffuse connective tissue disorders), M051 (rheumatoid lung disease), M313 (Wegener's granulomatosis), M321 (systemic lupus erythematosus with complications in organs or organ systems), M330 (juvenile dermatomyositis), M331 (other dermatomyositis), M332 (polymyositis) and M351 (other overlapping syndromes). Because corticosteroids are used to treat lung injury following DILD, ${ }^{1-11}$ we used the initiation of systemic corticosteroid therapy for treating lung injury following DILD as a proxy measure for the onset of DILD. Therefore, only patients with DILD who required corticosteroid therapy were selected as case patients in this study. Systemic corticosteroid therapy for treating lung injury following DILD was defined as follows: (1) high-dose corticosteroid therapy ( $>500 \mathrm{mg}$ of prednisolone per day for $\geq 3$ days); (2) medium-dose corticosteroid therapy $(201-500 \mathrm{mg}$ of prednisolone per day for $\geq 3$ days); and (3) low-dose corticosteroid therapy (30-200 mg of prednisolone per day for $\geq 7$ days). We evaluated the prescription duration of systemic corticosteroids for the case and control patients.

\section{Case-control matching}

From the entire cohort during the study period, four controls were matched to each case, using risk-set sampling with replacement. More specifically, for each patient with DILD, we selected four control patients with the same primary diagnosis (assessed using the first three digits of the patient's ICD-10 codes on admission), sex and age (within a 10-year difference) who were also hospitalised in the same hospital in the same fiscal year or consecutive fiscal years. In the risk-set sampling, control patients were selected from patients whose length of stay was longer than the duration from admission to the initiation of corticosteroid therapy in the corresponding case patients. Control patients were also selected from patients with DILD who received corticosteroid therapy initiated later than this therapy was initiated for the corresponding case patients. We excluded case patients for whom no matched control patient was found. When more than four control patients were eligible to be matched to a case patient, we randomly selected four control patients. We omitted case-control patient groups when case patients had corticosteroid therapy initiated on the day of admission.

\section{Definition of covariates}

Based on Quan et al's ${ }^{14}$ algorithm, ICD-10 codes for comorbidities were converted to a score, and the sum of these scores was used to calculate the Charlson Comorbidity Index. We assessed the existence of a cancer diagnosis, including lung cancer (ICD-10 code: C34) and other cancers. Activities of daily living on admission were examined using the Barthel index $(0-100) .{ }^{15}$ We categorised the Barthel index as $0-90$ or $95-100$. The Brinkman index was calculated as the number of cigarettes smoked per day multiplied by the number of years the patient smoked. We categorised the Brinkman index as <200, 200-599 or $\geq 600$. Body mass index was categorised into the following three groups: $<18.5,18.5-24.9$ or $\geq 25.0 \mathrm{~kg} / \mathrm{m}^{2}$. All missing values on categorical variables were categorised as missing. We evaluated the severity of patients at hospitalisation by assessing whether they were admitted to an intensive care unit within 2 days of hospitalisation or received mechanical ventilation within 2 days of hospitalisation.

\section{Definition and classification of drugs with potential risk}

We identified drugs that were potentially related to DILD using the information provided in the product package insert from the Pharmaceuticals and Medical Devices Agency (http://www. pmda.go.jp/PmdaSearch/iyakuSearch/), which reported 323 generic names and 5726 trade names of drugs that may cause interstitial pneumonia as an adverse drug reaction. We classified the identified drugs into 75 categories on the basis of their function and mechanism of action. Of these categories, we omitted 33 in which none of the drugs was referred to in any of the five review articles on DILD, including a recent systematic review, ${ }^{1-5}$ or population-based cohort study. ${ }^{6}$ We thus evaluated 42 drug categories with 216 generic names that are generally accepted as drugs with potential risk of DILD (online supplemental e-Table 1).

We examined the use of these drugs among all case and control patients. The number of drug categories with potential risk prescribed for each patient was also assessed. Corticosteroids prescribed before drugs with potential risk may have modulated the clinical course of patients with potential to develop DILD. Thus, when any of the candidate drugs in these drug categories were prescribed to a patient after prescription of systemic corticosteroids, regardless of the prescribed dose and duration, these drugs were not regarded as candidates for drugs with potential risk of DILD. Furthermore, drugs that were prescribed within 3 days before discharge for patients who were discharged alive were not considered drugs with potential risk.

\section{Statistical analysis}

The demographic characteristics of the patients are expressed as numbers and percentages. Standardised mean difference was used to evaluate the balance of background characteristics between the case and control patients ${ }^{16}$ and an absolute value of $<0.1$ was considered well balanced. ORs and 95\% CIs of each drug category were calculated using a univariate conditional logistic regression model. We selected drug categories that were significantly associated with DILD in the univariate analyses. We then performed a multivariable conditional logistic regression analysis with selected drug categories. We also included the Barthel index, Brinkman index, body mass index, Charlson Comorbidity Index, presence of lung cancer, presence of other cancer, intensive care unit admission within 2 days of hospitalisation and mechanical ventilation within 2 days of hospitalisation as independent variables in the model. Because the Barthel 
index, Brinkman index and body mass index had missing values, we performed multiple imputation for conditional multivariable logistic regression analysis. In brief, we used in-hospital mortality, age, sex, fiscal year, length of stay, diagnosis of lung cancer, diagnosis of malignancy other than lung cancer, intensive care unit admission during hospitalisation, mechanical ventilation during hospitalisation and all drug categories used for $\geq 5$ patients (online supplemental e-Table 2) for multivariate imputation by chained equation, and 20 imputed data sets were obtained. These data sets were then combined using Rubin's rule to acquire combined imputation estimates and SEs. ${ }^{17}$

A $\mathrm{p}$ value $<0.05$ was considered significant. Statistical analyses were performed using SPSS V.25.0 and Stata V.14.

\section{RESULTS}

We identified 2342 cases who developed DILD requiring corticosteroid therapy during hospitalisation in the study period. Four control patients were matched to each case patient from a total of 41771128 non-case hospitalised patients (online supplemental e-Figure 1). Of the cases, we excluded 292 that were not matched to any control patients. There were 118 cases that could be matched with only 1 control patient, 102 cases that could be matched with only 2 control patients, and 83 cases that could be matched with only 3 control patients.

We ultimately enrolled a total of 7218 patients, including 1541 cases and 5677 controls. Among the case patients, 1246 (81\%), 119 (7.7\%) and 671 (44\%) received high-dose, moderate-dose and low-dose corticosteroid therapy, respectively. The median ages of the case and control patients were 73 (IQR: 66-78) years and 73 (IQR: 66-79) years, respectively. Male patients made up $70 \%$ of both the case and the control group. The median length of hospital stay was $37(25-57)$ days for case patients and 20 (12-36) days for control patients. The median prescription duration of systemic corticosteroids was 29 (17-46) days for case patients and $9(3-26)$ days for control patients. The number (\%) of in-hospital deaths among the case and control patients was $535(35 \%)$ and $610(11 \%)$, respectively.

The characteristics, comorbidities, treatments at hospitalisation and number of prescribed drugs with potential risk of DILD of the case and control patients are shown in table 1 . Matched variables (age, sex and fiscal year) were well balanced between the cases and controls, except for fiscal years 2014 and 2015, which had standardised mean differences of 0.112 and 0.100 , respectively. The percentages of case and control patients requiring imputation due to missing data for activities of daily living, Brinkman index and body mass index were $13.8 \%$ and $11.9 \%, 10.5 \%$ and $9.7 \%$, and $5.0 \%$ and $6.1 \%$, respectively.

One or more of the drug categories with potential risk were prescribed for $56 \%$ of the case patients and $45 \%$ of the control patients. The number of patients who received agents with potential risk, including all drugs prescribed during hospitalisation and those prescribed before prescription of any corticosteroids, is presented in online supplemental e-Table 2. We excluded a total of 23 drug categories because small numbers of patients $(<5)$ were administered these types of drugs in either the case group or the control group (online supplemental e-Figure 2).

Approximately $6.8 \%$ of the control patients received corticosteroids equivalent to one or more instances of high-dose, medium-dose or low-dose corticosteroid therapy. Table 2 shows the results of the univariate conditional logistic regression analyses for treatment with systemic corticosteroids and in-hospital death. The percentage of patients treated with systemic corticosteroids was larger for case patients than for control patients, particularly for high-dose corticosteroid therapy $(80.9 \%$ vs $2.8 \%)$. Case patients had higher in-hospital mortality than did control patients (OR: 5.2, 95\% CI 4.49 to 6.10).

The number of patients who received agents with potential risk eligible for further analysis (ie, those that were not prescribed during the last 3 days of hospitalisation) and the results of the univariate conditional logistic regression analyses for the occurrence of DILD are shown in table 3. Of the 17 drug categories, 10 showed significant differences in the occurrence of DILD between cases and controls.

Table 4 shows the results of the multivariable conditional logistic regression analysis with multiple imputation including 10 drug categories. Higher Charlson Comorbidity Index, higher Brinkman index and lower body mass index were associated with an increased risk of developing DILD. Activities of daily living score was not significantly associated with the occurrence of DILD. Patients with lung cancer or other cancer had a higher incidence of DILD. Intensive care unit admission within 2 days of hospitalisation was not associated with an increased incidence of DILD, whereas patients who were mechanically ventilated within 2 days of hospitalisation had an increased incidence of DILD. Of the 10 drug categories, 6 were significantly associated with the development of DILD, including class III antiarrhythmic drugs, non-steroidal anti-inflammatory drugs (NSAIDs), sulfamethoxazole/trimethoprim, quinolones, betalactams and epidermal growth factor receptor (EGFR) inhibitors. The drug categories with OR $>5$ included EGFR inhibitors (OR: $16.84,95 \%$ CI 9.32 to 30.41 ) and class III antiarrhythmic drugs (OR: 7.01, 95\% CI 3.86 to 12.73 ). The drug categories with OR of 2-5 included sulfamethoxazole/trimethoprim and quinolones. Statins were the only drug category with an OR $<1$, and this association was statistically significant.

\section{DISCUSSION}

The present nested case-control study using a nationwide inpatient database investigated the use of 42 drug categories with potential risk in cases that developed DILD during hospitalisation and matched controls, finding significant associations between 6 drug categories and the occurrence of DILD.

We evaluated only drugs that were mentioned in previous review articles on DILD, ${ }^{1-6}$ and we used drug categories with similar biological activity for two reasons: (1) Evaluating each drug individually would increase the number of variables for drugs with potential risk, and each drug would have fewer patients to whom it was prescribed, potentially reducing statistical power. (2) Because some candidate drugs were domestic drugs, we used drug categories to avoid eliminating drugs due to the absence of previous reports in English. Nevertheless, we may still have missed some significant associations by eliminating drugs due to the absence of previous reports. However, the evaluated drugs can be considered core drugs for DILD because they are likely to be drugs with relatively high risk of developing DILD or with risk of developing severe DILD.

Previous studies have identified male sex, older age and preexisting lung disease as common risk factors of DILD caused by amiodarone, methotrexate and EGFR inhibitor. ${ }^{18-22}$ In line with these previous studies, in our study, DILD was more frequent in male patients and $88 \%$ of patients with DILD were aged $\geq 60$ years. Because multiple comorbidities may be a source of confounding (ie, patients with multiple comorbidities are likely to be exposed to multiple treatments, including drugs with potential risk, and may also be vulnerable to adverse events), we included the Charlson Comorbidity Index in our multivariable regression model. We also found 


\section{Interstitial lung disease}

Table 1 Patient characteristics of the matched case and control groups

\begin{tabular}{|c|c|c|c|c|c|}
\hline \multirow[b]{2}{*}{ Characteristics } & \multicolumn{2}{|c|}{ Cases $(n=1541)$} & \multicolumn{2}{|c|}{ Controls $(n=5677)$} & \multirow[b]{2}{*}{ SMD } \\
\hline & $\mathrm{n}$ & $\%$ & $\mathrm{n}$ & $\%$ & \\
\hline \multicolumn{6}{|l|}{ Age, years } \\
\hline$\leq 10$ & 0 & 0.0 & 0 & 0.0 & 0.005 \\
\hline $11-20$ & 1 & 0.1 & 3 & 0.1 & -0.003 \\
\hline $21-30$ & 2 & 0.1 & 8 & 0.1 & 0.017 \\
\hline $31-40$ & 8 & 0.5 & 23 & 0.4 & 0.048 \\
\hline $41-50$ & 38 & 2.5 & 101 & 1.8 & 0.024 \\
\hline $51-60$ & 137 & 8.9 & 467 & 8.2 & -0.027 \\
\hline $61-70$ & 447 & 29.0 & 1717 & 30.2 & 0.061 \\
\hline $71-80$ & 647 & 42.0 & 2215 & 39.7 & -0.073 \\
\hline $81-90$ & 248 & 16.1 & 1071 & 18.9 & -0.042 \\
\hline$\geq 91$ & 15 & 0.8 & 72 & 1.3 & \\
\hline Sex & & & & & 0.010 \\
\hline Male & 1079 & 70.0 & 4000 & 70.5 & \\
\hline Female & 462 & 30.0 & 1677 & 29.5 & \\
\hline \multicolumn{6}{|l|}{ Fiscal year } \\
\hline 2010 & 92 & 6.0 & 330 & 5.8 & 0.009 \\
\hline 2011 & 183 & 11.9 & 701 & 12.3 & -0.014 \\
\hline 2012 & 293 & 19.0 & 1069 & 18.8 & 0.005 \\
\hline 2013 & 312 & 20.3 & 1087 & 19.2 & 0.028 \\
\hline 2014 & 307 & 19.9 & 1420 & 25.0 & 0.112 \\
\hline 2015 & 353 & 22.9 & 1070 & 18.9 & 0.100 \\
\hline \multicolumn{6}{|l|}{ Activities of daily living (Barthel index) } \\
\hline $95-100$ & 819 & 53.1 & 3051 & 53.7 & -0.012 \\
\hline $0-90$ & 509 & 33.0 & 1951 & 34.4 & -0.028 \\
\hline Missing & 213 & 13.8 & 675 & 11.9 & 0.058 \\
\hline \multicolumn{6}{|l|}{ Brinkman index } \\
\hline 0-199 & 712 & 46.2 & 3606 & 63.5 & -0.353 \\
\hline 200-599 & 164 & 10.6 & 483 & 8.5 & 0.073 \\
\hline$\geq 600$ & 504 & 32.7 & 1036 & 18.3 & 0.336 \\
\hline Missing & 161 & 10.5 & 552 & 9.7 & 0.024 \\
\hline \multicolumn{6}{|l|}{ Body mass index, $\mathrm{kg} / \mathrm{m}^{2}$} \\
\hline$<18.50$ & 247 & 16.0 & 1233 & 21.7 & -0.146 \\
\hline $18.50-24.99$ & 1005 & 65.2 & 3132 & 55.2 & 0.206 \\
\hline$\geq 25.00$ & 212 & 13.8 & 964 & 17.0 & -0.089 \\
\hline Missing & 77 & 5.0 & 348 & 6.1 & -0.049 \\
\hline \multicolumn{6}{|l|}{ Charlson Comorbidity Index } \\
\hline $0-2$ & 983 & 63.8 & 4312 & 76.0 & -0.268 \\
\hline $3-5$ & 105 & 6.8 & 356 & 6.3 & 0.022 \\
\hline$\geq 6$ & 453 & 29.4 & 1009 & 17.8 & 0.278 \\
\hline \multicolumn{6}{|l|}{ Cancer } \\
\hline Lung cancer & 555 & 36.0 & 1725 & 30.4 & 0.120 \\
\hline Other cancer & 481 & 31.2 & 1514 & 26.7 & 0.100 \\
\hline ICU admission within 2 days of hospitalisation & 55 & 3.6 & 261 & 4.6 & -0.052 \\
\hline Mechanical ventilation within 2 days of hospitalisation & 74 & 4.8 & 219 & 3.9 & 0.046 \\
\hline
\end{tabular}

$\mathrm{ICU}$, intensive care unit; SMD, standardised mean difference.

that having a lower body mass index and having a higher Charlson Comorbidity Index score were significant independent risk factors for the development of DILD. A previous study reported that lung cancer was a strong predictor of ILD associated with gemcitabine. ${ }^{23}$ In line with this previous study, lung cancer was found to be a significant risk factor for DILD in our study. 
Table 2 Comparison of systemic corticosteroid treatments and in-hospital death between case and control patients using univariate conditional logistic regression analyses

\begin{tabular}{|c|c|c|c|c|c|c|c|}
\hline \multirow[b]{2}{*}{ Drug category with potential risk } & \multicolumn{2}{|c|}{$\begin{array}{l}\text { Cases } \\
(n=1541)\end{array}$} & \multicolumn{2}{|c|}{$\begin{array}{l}\text { Controls } \\
(n=5677)\end{array}$} & \multirow[b]{2}{*}{ OR } & \multirow[b]{2}{*}{$95 \% \mathrm{Cl}$} & \multirow[b]{2}{*}{$P$ value* } \\
\hline & $\mathbf{n}$ & $\%$ & $\mathbf{n}$ & $\%$ & & & \\
\hline High-dose corticosteroid therapy & 1246 & 80.9 & 156 & 2.8 & 185.92 & 123.0 to 280.9 & $<0.001$ \\
\hline Moderate-dose corticosteroid therapy & 119 & 7.7 & 28 & 0.5 & 19.30 & 12.10 to 30.80 & $<0.001$ \\
\hline Low-dose corticosteroid therapy & 671 & 43.5 & 263 & 4.6 & 18.47 & 15.17 to 22.48 & $<0.001$ \\
\hline \multicolumn{8}{|l|}{ Outcome of DILD } \\
\hline In-hospital death & 535 & 34.7 & 610 & 10.8 & 5.24 & 4.49 to 6.10 & $<0.001$ \\
\hline
\end{tabular}

*Denotes $\mathrm{p}$ values derived from univariate conditional logistic regression.

DILD, drug-induced interstitial lung disease.

To the best of our knowledge, no previous large-scale study has shown the differences in risk among drugs that are likely to be responsible for DILD. Drugs with potential risk were prescribed for $56 \%$ of the cases. Presumably, in the other $44 \%$ of the cases, either DILD was caused by drugs that were not included in our study or delayed-onset DILD caused by drugs with potential risk prescribed in outpatient settings developed during hospitalisation. The lack of data in outpatient settings may also limit the applicability of our findings because hospitalised patients are generally sicker and may have higher risk of development of DILD, compared with outpatients. Nevertheless, our study showed that EGFR inhibitors and class III antiarrhythmic drugs were highly likely to lead to the development of DILD (OR $\geq 5)$.

Our results support the findings of previous small cohort studies and case reports. EGFR showed the highest OR among all drug categories included in this study. A previous cohort study reported that the risk of developing DILD among patients with lung cancer was generally higher in patients taking gefitinib than in patients receiving cytotoxic chemotherapy, particularly in the first 4 weeks. ${ }^{21}$ A class III antiarrhythmic drug (amiodarone) was found to be associated with acute lung injury in a recent retrospective cohort study. ${ }^{6}$ However, to date, no large cohort study has explored the effects of these drugs.

Our findings also support the results of previous reports that have suggested a potential risk of interstitial pneumonia in patients receiving a quinolone,$^{24-26}$ which is thought to potentially be associated with the risk of developing DILD.

Although cases of DILD caused by NSAIDs ${ }^{2} 27$ and betalactams ${ }^{2528}$ have previously been reported and these drugs were significantly associated with increased incidence of DILD in our study, the ORs of these drug categories were $<2$. Despite the statistical significance of these associations, the clinical impact of drugs with relatively low ORs may be limited. However, because these drugs are commonly prescribed and the key to the successful treatment of DILD is early recognition of drugs with potential risk, care should be taken when using these drugs in patients who develop DILD.

Table 3 Drug categories with potential risk of drug-induced interstitial lung disease prescribed before corticosteroids and $\leq 3$ days before discharge during hospitalisation and the results of univariate conditional logistic regression analyses for the occurrence of drug-induced interstitial lung disease

\begin{tabular}{|c|c|c|c|c|c|c|c|}
\hline \multirow[b]{2}{*}{ Drug category with potential risk } & \multicolumn{2}{|c|}{ Cases $(n=1541)$} & \multicolumn{2}{|c|}{ Controls $(n=5677)$} & \multirow[b]{2}{*}{ OR } & \multirow[b]{2}{*}{$95 \% \mathrm{Cl}$} & \multirow[b]{2}{*}{$P$ value } \\
\hline & $\mathbf{n}$ & $\%$ & $\mathbf{n}$ & $\%$ & & & \\
\hline Antiplatelets & 44 & 2.9 & 150 & 2.6 & 1.01 & 0.69 to 1.46 & 0.977 \\
\hline Anticoagulants & 7 & 0.5 & 9 & 0.2 & 3.11 & 1.16 to 8.35 & 0.024 \\
\hline Statins & 60 & 3.9 & 294 & 5.2 & 0.68 & 0.50 to 0.92 & 0.012 \\
\hline Sodium channel blockers & 10 & 0.6 & 30 & 0.5 & 1.28 & 0.61 to 2.67 & 0.511 \\
\hline Class III antiarrhythmic drugs & 45 & 2.9 & 35 & 0.6 & 6.80 & 3.96 to 11.66 & $<0.001$ \\
\hline ACE inhibitor & 9 & 0.6 & 18 & 0.3 & 1.83 & 0.80 to 4.20 & 0.156 \\
\hline Thiazides & 11 & 0.7 & 67 & 1.2 & 0.59 & 0.31 to 1.14 & 0.116 \\
\hline NSAIDs & 302 & 19.6 & 566 & 10.0 & 2.43 & 2.04 to 2.88 & $<0.001$ \\
\hline Antiepileptics & 16 & 1.0 & 53 & 0.9 & 1.07 & 0.59 to 1.94 & 0.836 \\
\hline Sulfamethoxazole/trimethoprim & 14 & 0.9 & 17 & 0.3 & 2.89 & 1.36 to 6.13 & 0.006 \\
\hline Quinolones & 175 & 11.4 & 246 & 4.3 & 2.93 & 2.35 to 3.65 & $<0.001$ \\
\hline Tetracyclines & 40 & 2.6 & 69 & 1.2 & 2.30 & 1.50 to 3.52 & $<0.001$ \\
\hline Beta-lactams & 639 & 41.5 & 1940 & 34.2 & 1.62 & 1.39 to 1.89 & $<0.001$ \\
\hline Anti-TB drugs & 14 & 0.9 & 28 & 0.5 & 2.14 & 0.94 to 4.89 & 0.071 \\
\hline EGFR inhibitors & 73 & 4.7 & 22 & 0.4 & 16.48 & 9.57 to 28.39 & $<0.001$ \\
\hline Pyrimidine & 16 & 1.0 & 50 & 0.9 & 1.18 & 0.63 to 2.21 & 0.599 \\
\hline Anthracyclines & 9 & 0.6 & 12 & 0.2 & 3.09 & 1.24 to 7.70 & 0.015 \\
\hline
\end{tabular}

EGFR, epidermal growth factor receptor; NSAIDs, non-steroidal anti-inflammatory drugs. 
Table 4 Multivariable conditional logistic regression analysis for the occurrence of drug-induced interstitial lung disease

\begin{tabular}{|c|c|c|c|}
\hline Characteristics & OR & $95 \% \mathrm{Cl}$ & $P$ value \\
\hline \multicolumn{4}{|l|}{ Barthel index } \\
\hline $95-100$ & Reference & & \\
\hline $0-90$ & 0.93 & 0.78 to 1.09 & 0.352 \\
\hline \multicolumn{4}{|l|}{ Brinkman index } \\
\hline 0-199 & Reference & & \\
\hline $200-599$ & 1.64 & 1.31 to 2.05 & $<0.001$ \\
\hline$\geq 600$ & 2.54 & 2.17 to 2.98 & $<0.001$ \\
\hline \multicolumn{4}{|l|}{ Body mass index } \\
\hline$<18.50$ & 1.65 & 1.38 to 1.96 & $<0.001$ \\
\hline $18.50-24.99$ & Reference & & \\
\hline$\geq 25.00$ & 1.18 & 0.93 to 1.48 & 0.169 \\
\hline \multicolumn{4}{|l|}{ Charlson Comorbidity Index } \\
\hline $0-2$ & Reference & & \\
\hline $3-5$ & 1.01 & 0.77 to 1.32 & 0.955 \\
\hline$\geq 6$ & 2.39 & 2.00 to 2.86 & $<0.001$ \\
\hline Lung cancer & 2.38 & 1.81 to 3.12 & $<0.001$ \\
\hline Other cancer (other than lung cancer) & 1.77 & 1.43 to 2.20 & $<0.001$ \\
\hline $\begin{array}{l}\text { ICU admission within } 2 \text { days of } \\
\text { hospitalisation }\end{array}$ & 0.68 & 0.46 to 1.02 & 0.063 \\
\hline $\begin{array}{l}\text { Mechanical ventilation within } 2 \text { days } \\
\text { of hospitalisation }\end{array}$ & 1.77 & 1.22 to 2.47 & 0.002 \\
\hline \multicolumn{4}{|l|}{ Drug category with potential risk } \\
\hline Anticoagulants & 2.58 & 0.76 to 8.81 & 0.130 \\
\hline Statins & 0.53 & 0.37 to 0.75 & $<0.001$ \\
\hline Class III antiarrhythmic drugs & 7.01 & 3.86 to 12.73 & $<0.001$ \\
\hline NSAIDs & 1.90 & 1.56 to 2.31 & $<0.001$ \\
\hline Sulfamethoxazole/trimethoprim & 2.54 & 1.04 to 6.24 & 0.042 \\
\hline Quinolones & 3.10 & 2.41 to 3.99 & $<0.001$ \\
\hline Tetracyclines & 1.60 & 0.97 to 2.66 & 0.067 \\
\hline Beta-lactams & 1.54 & 1.29 to 1.84 & $<0.001$ \\
\hline EGFR inhibitors & 16.84 & 9.32 to 30.41 & $<0.001$ \\
\hline Anthracyclines & 1.89 & 0.68 to 5.23 & 0.223 \\
\hline
\end{tabular}

Drug categories with potential risk of drug-induced interstitial lung disease with significant associations in the univariate analysis were included.

EGFR, epidermal growth factor receptor; ICU, intensive care unit; NSAIDs, nonsteroidal anti-inflammatory drugs.

The only drug category with an OR $<1$ in this study was statins. Statin-induced pneumonitis is well recognised, ${ }^{1-3} 2930$ and the protective effect of statins in cases of lung disease, such as in acute lung injury, ${ }^{31}$ radiation pneumonitis ${ }^{32}$ and interstitial pneumonia, ${ }^{33}$ has been discussed with mixed results. Two large-scale, nested case-control studies using insurance claims databases specifically evaluated the association between statins and ILD. A study conducted in Quebec found no association between the development of ILD and statins, ${ }^{34}$ whereas a study in Japan reported a significant association between ILD and atorvastatin. ${ }^{35}$ Our study has three potential advantages over these two studies. First, our study included only patients who were diagnosed during hospitalisation and were treated with corticosteroids; therefore, our study may have included patients with DILD with higher specificity and severity. Second, we assessed the discontinuation of drugs with potential risk; therefore, the drugs assessed in our study are more likely to be a risk. Third, in our study, risk factors were adjusted for various drugs with potential risk, allowing us to evaluate the independent risk of each drug category for patients using multiple drugs with potential risk. The exclusion of patients with mild DILD who recovered without the use of systemic corticosteroids may have biased our results. However, there is no evidence that DILD caused by statins is generally milder than DILD caused by other drugs. We speculate that statins are associated with only a modest risk of developing DILD requiring corticosteroid therapy, relative to the risk of other drugs prescribed during hospitalisation. Further studies are required to confirm our results.

The present study also did not find significant associations between many drug categories and DILD. However, this finding should be interpreted carefully, and we cannot conclude these drugs are safe because previous studies have suggested that most of these drug categories were potential risks. These drugs may have caused DILD in patients who recovered after discontinuing use of the drugs, and thus the risks associated with these drugs were not accurately evaluated in this study. In addition, the lack of significant findings in our study may simply reflect insufficient statistical power for infrequently used drugs.

The pre-existence of ILD and prior or concurrent use of corticosteroids may have been candidates for effect modifiers if they had been included in our study. We excluded patients who were diagnosed with ILD on admission for two reasons. First, ILD may also be a confounder due to its association with certain drugs evaluated in this study. For example, ILD is likely to be associated with both the use of EGFR inhibitors and the outcome. Second, distinguishing the development of DILD from the acute exacerbation of ILD may be difficult. Drugs used after systemic corticosteroids were excluded from the list of drugs with potential risk in this study. Corticosteroids are used for the treatment of severe DILD and therefore might modify the effects of exposure to the evaluated drugs on risk of DILD. Although we did not explore potential effect modifiers or interactions between drugs with potential risk of DILD in this study, our study will help foster future investigation.

The limitations of this study should be acknowledged. First, from the information in the database, we were unable to identify the exact date of the onset of DILD during hospitalisation. Thus, we were not able to definitely confirm that the drugs were used before the onset of DILD. We used the date of systemic corticosteroid treatment as a proxy for the date of the development of DILD. We were therefore unable to evaluate mild DILD from which patients recovered after discontinuing drugs, without systemic corticosteroid therapy. Second, in this study setting, we were unable to assess drugs such as cisplatin, which are coadministered with corticosteroids to prevent adverse effects. Third, we did not explore statistical interactions between the drugs. Fourth, several types of clinical data were not available in the database, including results of chest X-rays, high-resolution CT, pathology and blood testing. Severity, pathological manifestation and radiological phenotype of DILD could not be assessed. Fifth, we could not evaluate drug categories prescribed for a very small number of patients. Finally, this study was prone to confounding by indication because this was a retrospective database study.

In conclusion, we conducted a nested case-control study to identify significant associations between several drug categories and the occurrence of DILD in hospitalised patients who required corticosteroid therapy, using a national inpatient database. Our findings may provide useful information on drugs with potential risk of DILD, assisting physicians in the prevention and treatment of DILD. 
Contributors TJ: conception and design, data analysis and interpretation, and manuscript writing. NM, HYam, YY, WH, UH: conception and design, data analysis and interpretation, and final approval of the manuscript. KM, MI, KU, KF, HM: data collection, data analysis and final approval of the manuscript. HYas: conception and design, data collection, data analysis and interpretation, and manuscript writing. TN conception and design and final approval of the manuscript.

Funding This work was supported by grants from the Ministry of Health, Labour and Welfare, Japan (19AA2007 and H30-Policy-Designated-004) and the Ministry of Education, Culture, Sports, Science and Technology, Japan (17H04141).

Competing interests None declared.

Patient consent for publication Not required.

Ethics approval The present study was approved by the Institutional Review Board of The University of Tokyo, which waived the requirement for patient informed consent due to the anonymous nature of the data.

Provenance and peer review Not commissioned; externally peer reviewed.

Data availability statement Data are available upon reasonable request. Data cannot be made publicly available for ethical reasons as the data are patient data. Data are available to interested researchers upon reasonable request to the corresponding author, pending ethical approval.

\section{REFERENCES}

1 Skeoch S, Weatherley N, Swift A, et al. Drug-Induced interstitial lung disease: a systematic review. JCM 2018;7:356.

2 Prasad R, Gupta P, Singh A, et al. Drug induced pulmonary parenchymal disease. Drug Discov Ther 2014;8:232-7.

3 Schwaiblmair M, Behr W, Haeckel T, et al. Drug induced interstitial lung disease. Open Respir Med J 2012;6:63-74

4 Cooper JA, White DA, Matthay RA. Drug-induced pulmonary disease. Part 1: cytotoxic drugs. Am Rev Respir Dis 1986;133:321-40.

5 Cooper JA, White DA, Matthay RA. Drug-induced pulmonary disease. Part 2: noncytotoxic drugs. Am Rev Respir Dis 1986;133:488-505.

6 Dhokarh R, Li G, Schmickl CN, et al. Drug-associated acute lung injury: a populationbased cohort study. Chest 2012;142:845-50.

7 Kataoka K, Taniguchi H, Hasegawa Y, et al. Interstitial lung disease associated with gefitinib. Respir Med 2006;100:698-704.

8 Wong PW, Macris N, DiFabrizio L, et al. Eosinophilic lung disease induced by bicalutamide: a case report and review of the medical literature. Chest 1998; 113:548-50

9 Vahid B, Marik PE. Pulmonary complications of novel antineoplastic agents for solid tumors. Chest 2008;133:528-38.

10 Camus $\mathrm{P}$, Kudoh S, Ebina M. Interstitial lung disease associated with drug therapy. $\mathrm{Br} J$ Cancer 2004;91 Suppl 2:S18-23.

11 Müller NL, White DA, Jiang $H$, et al. Diagnosis and management of drug-associated interstitial lung disease. Br J Cancer 2004;91 Suppl 2:S24-30.

12 Suzuki Y, Miwa S, Shirai M, et al. Drug lymphocyte stimulation test in the diagnosis of adverse reactions to antituberculosis drugs. Chest 2008;134:1027-32.

13 Hirata S, Hattori N, Kumagai K, et al. Lymphocyte transformation test is not helpful for the diagnosis of methotrexate-induced pneumonitis in patients with rheumatoid arthritis. Clin Chim Acta 2009;407:25-9.
14 Quan H, Li B, Couris CM, et al. Updating and validating the Charlson comorbidity index and score for risk adjustment in hospital discharge Abstracts using data from 6 countries. Am J Epidemiol 2011;173:676-82.

15 Mahoney FI, Barthel DW. Functional evaluation: the Barthel index. Md State Med J 1965;14:61-5.

16 Miyamoto Y, Iwagami M, Aso S, et al. Pregabalin and injury: a nested case-control and case-crossover study. Pharmacoepidemiol Drug Saf 2020;29:558-64.

17 Aloisio KM, Swanson SA, Micali N, et al. Analysis of partially observed clustered data using generalized estimating equations and multiple imputation. Stata J 2014;14:863-83.

18 Wolkove N, Baltzan M. Amiodarone pulmonary toxicity. Can Respir J 2009;16:43-8.

19 Kudoh S, Kato H, Nishiwaki Y, et al. Interstitial lung disease in Japanese patients with lung cancer: a cohort and nested case-control study. Am J Respir Crit Care Med 2008; 177:1348-57.

20 Shidara K, Hoshi D, Inoue E, et al. Incidence of and risk factors for interstitial pneumonia in patients with rheumatoid arthritis in a large Japanese observational cohort, IORRA. Mod Rheumatol 2010:20:280-6.

21 Ando $\mathrm{M}, \mathrm{Okamoto} \mathrm{I}$, Yamamoto $\mathrm{N}$, et al. Predictive factors for interstitial lung disease, antitumor response, and survival in non-small-cell lung cancer patients treated with gefitinib. J Clin Oncol 2006;24:2549-56.

22 Ohosone $\mathrm{Y}$, Okano Y, Kameda H, et al. Clinical characteristics of patients with rheumatoid arthritis and methotrexate induced pneumonitis. J Rheumatol 1997;24:2299-303.

23 Hamada T, Yasunaga H, Nakai Y, et al. Interstitial lung disease associated with gemcitabine: a Japanese retrospective cohort study. Respirology 2016;21:338-43.

24 Steiger D, Bubendorf L, Oberholzer M, et al. Ciprofloxacin-induced acute interstitial pneumonitis. Eur Respir J 2004;23:172-4.

25 Facciolongo N, Menzella F, Castagnetti C, et al. Eosinophilic infiltrate in a patient with severe Legionella pneumonia as a levofloxacin-related complication: a case report. $J$ Med Case Rep 2010;4:360.

26 Hosogaya $\mathrm{N}$, Toida $\mathrm{K}$, Ishihara $\mathrm{H}$, et al. A case of drug induced lung injury caused by levofloxacin eye drops. Respir Med Case Rep 2018;24:12-15.

27 Ueda K, Sakano H, Tanaka T, et al. Diclofenac (Voltaren)-induced pneumonitis after chest operation. Ann Thorac Surg 2002;74:2176-7.

28 Tseng OL-I, Kelsall JT, Wilcox PG. Piperacillin-associated pulmonary infiltrates with eosinophilia: a case report. Can Respir J 2010;17:e24-6.

29 Lantuejoul S, Brambilla E, Brambilla C, et al. Statin-induced fibrotic nonspecific interstitial pneumonia. Eur Respir J 2002;19:577-80.

30 Fernández AB, Karas RH, Alsheikh-Ali AA, et al. Statins and interstitial lung disease: a systematic review of the literature and of food and drug administration adverse event reports. Chest 2008;134:824-30.

31 Xiong B, Wang C, Tan J, et al. Statins for the prevention and treatment of acute lung injury and acute respiratory distress syndrome: a systematic review and meta-analysis. Respirology 2016;21:1026-33.

32 Vasiljevic D, Arnold C, Neuman D, et al. Occurrence of pneumonitis following radiotherapy of breast cancer - A prospective study. Strahlenther Onkol 2018;194:520-32.

33 Vedel-Krogh S, Nielsen SF, Nordestgaard BG. Statin use is associated with reduced mortality in patients with interstitial lung disease. PLoS One 2015;10:e0140571.

34 Saad N, Camus P, Suissa S, et al. Statins and the risk of interstitial lung disease: a cohort study. Thorax 2013;68:361-4.

35 Momo K, Takagi A, Miyaji A, et al. Assessment of statin-induced interstitial pneumonia in patients treated for hyperlipidemia using a health insurance claims database in Japan. Pulm Pharmacol Ther 2018;50:88-92. 